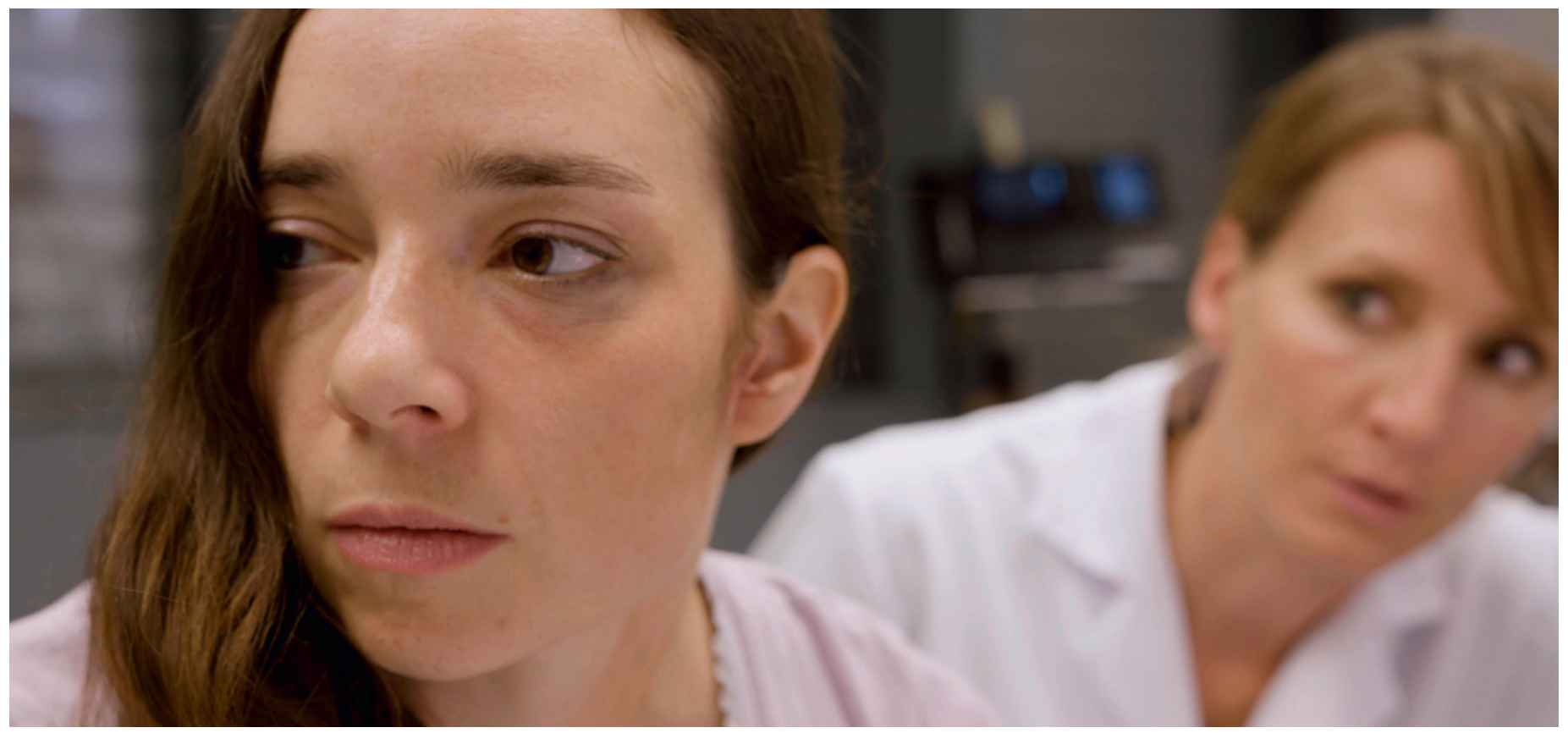

\title{
Et si cette patiente était victime de traite d'êtres humains?
}

\section{Anne-Florence Débois}

Porte-parole de fedpol, Office fédéral de la police

C’est une réalité en Suisse. En 2017, 149 personnes ont été exploitées sexuellement ou pour leur force de travail. Subissant des violences physiques, elles sont parfois amenées par leur bourreau à l'hôpital. En tant que professionnels de la santé, vous jouez un rôle décisif lors de la consultation. fedpol lance une campagne de sensibilisation pour que vous puissiez les reconnaître et les aider.

Aucune marque de coups n'est visible sur son visage. Les impressionnants hématomes au bas de son dos virent au bleu. La patiente a aussi des ecchymoses sur le ventre, ils ont depuis jaunis. Après l'analyse de son urine, elle souffre également d'une grave infection urinaire. A première vue, difficile de savoir ce qui amène cette jeune femme à l'hôpital. Elle ne parle pas français, mais un homme qui dit être son compagnon a insisté pour rester avec elle durant la consultation.

Cette femme est en réalité une victime de traite d'être humain. Elle est exploitée sexuellement, a été battue il y a plusieurs jours déjà. Chaque soir, elle subit des me- naces si elle refuse de se prostituer dans la rue. Cette fois-ci, son proxénète s'en est violemment pris à elle, la ruant de coups dans le dos parce qu'elle ne lui a pas

Cette femme est en réalité une victime de traite d'être humain. Elle est exploitée sexuellement.

rapporté assez d'argent. C'est sans compter le traumatisme d'avoir quitté sa famille, ses parents il y a plusieurs mois déjà. Elle avait l'espoir de fonder une nouvelle vie en Suisse à l'abri du besoin et d'y trouver un vrai travail. 
En tant que médecin, en tant qu'aide-soignant, en tant qu'assistante médicale, auriez-vous reconnu qu'il s'agit là d'une victime de traite d'être humain? Comment pourriez-vous l'aider à fuir l'emprise de son bourreau? Que pouvez-vous faire concrètement pour elle?

\section{Vous avez un rôle décisif à jouer}

Fin juin, à l'Hôpital de l'Ile à Berne, fedpol a lancé une campagne nationale de sensibilisation auprès du personnel médical et soignant. Cette campagne cherche à éveiller les consciences sur un sujet qui reste tabou, mais qui ne devrait pas être ignoré. La Suisse est concernée par la traite des êtres humains. C'est un fait: les trafiquants accompagnent les victimes blessées ou en mauvaise santé à l'hôpital. Ils ont un intérêt à ce qu'elles reçoivent des soins médicaux et ils privilégient les grands hôpitaux ou les centres médicaux où ils ne courent pas le risque de se faire remarquer. Dans la lutte contre la traite des êtres humains, une consultation à l'hôpital ou aux urgences représente un premier filtre pour pouvoir identifier ces victimes. C'est surtout une chance pour elles de s'en sortir.

En tant que professionnels de la santé, vous avez un rôle décisif à jouer. Cette campagne a pour but de vous aider à reconnaître les patientes et patients qui pourraient être victimes de traite.

\section{Chaque cas est différent}

Il est difficile de repérer une victime de traite. Les signes de détresse ne sont pas reconnaissables au premier coup d'œil, ils s'expriment de façon subtile. Le cas type n'existe pas, chaque cas est différent. Il s'agit de savoir interpréter à la fois des indices physiques, psychiques et d'ordre contextuel. Par exemple, une femme est accompagnée d'un homme dont le comportement

\section{Il est difficile de repérer une victime de traite.}

est agressif et qui insiste pour rester pendant la consultation. La patiente s'exprime dans une langue étrangère, elle a un regard fuyant ou craintif. Les raisons exactes des blessures sur son corps sont expliquées de manière insatisfaisante.

Ces indices possibles sont présentés dans un flyer distribué aux hôpitaux et aux cabinets médicaux. L'objectif est de permettre aux professionnels de la santé de mieux reconnaître les victimes de la traite, afin de les protéger et les aider. Un court-métrage accompagne ce flyer. Il montre la difficulté d'aider une victime de traite.

\section{Que puis-je faire concrètement?}

Si vous décelez une situation délicate, n'hésitez pas à agir. Sachez que les victimes de traite d'êtres humains sont vulnérables et fragiles psychologiquement. Elles se méfient souvent des autorités par peur de représailles si, par exemple, elles parlent à la police. Une première aide peut être la simple transmission d'un contact avec une association d'aide aux victimes. Une pocketcard avec un numéro d'urgence a été produite à cet effet. Vous pouvez donner cette carte à la victime qui pourra décider, dans un deuxième temps, si elle souhaite demander de l'aide.

Dans le pire des cas, la patiente se refuse à vous répondre ou vous donne des informations confuses par rapport à ces blessures physiques. Vous pouvez lui faire comprendre qu'elle peut se sortir de la situation et lui donner la pocketcard en lui disant par exemple: "J'ai bien compris que vous n'êtes pas en mesure de faire quoi que ce soit aujourd'hui, mais prenez cette information, au cas où."

Dans le meilleur des cas, la patiente est réceptive. Vous pouvez lui expliquer qu'elle a des droits et que vous pouvez la mettre en contact avec une association d'aide aux victimes ou avec les autorités de polices cantonales. Pour contacter ces instances, vous devez obtenir le consentement de la patiente.

\section{Gagnez du temps et la confiance du patient ou de la patiente}

Les professionnels de la santé sont aptes à faire face à ce genre de situation, mais ce n'est pas toujours facile dans le quotidien des urgences d'avoir les réflexes adéquats. Cela demande de l'expérience, la capacité de déceler l'indicible. Durant une consultation, le professionnel de la santé ne doit pas hésiter à poser beaucoup de questions et à chercher à en savoir le plus possible. Il peut faire appel à un service d'interprétariat si la patiente parle une langue étrangère. L'une des clés est de gagner du temps et la confiance de la victime pour pouvoir l'informer (voir encadré).

\section{Que va-t-il advenir de la victime?}

Une personne victime de traite d'êtres humains en Suisse a des droits et peut les faire valoir. Elle a droit à un soutien et à de l'aide, quels que soient sa nationalité et son statut de séjour. Elle a droit à être protégée, notamment à retrouver un logement ou à bénéficier d'un soutien sur le plan médical et juridique. Une fois le contact établi avec une association, tout va être mis en œuvre pour protéger la victime et faire en sorte de stabiliser sa situation, notamment si la victime a séjourné illégalement en Suisse. Si la victime est prête à le faire, elle peut dénoncer son bourreau et témoigner contre lui auprès de la police. Durant l'enquête, la victime peut obtenir une autorisation de séjour et bénéficie d'une protection durant et après la fin de la procédure pénale. 


\section{La Suisse aussi concernée}

En Suisse, en 2017, on compte 149 victimes identifiées. La traite existe sous différentes formes et dans différents domaines. La prostitution forcée est la plus répandue. Les victimes sont de jeunes femmes, souvent originaires du Sud-Est de l'Europe. La traite existe aussi sous la forme d'exploitation de la force du travail. Selon

Une personne victime de traite d'êtres humains en Suisse a des droits et peut les faire valoir.

l'étude «Exploitation du travail dans le contexte de la traite des êtres humains», publiée en 2016 par l'Université de Neuchâtel, les experts estiment que le nombre de cas non recensés reste élevé. Ils suspectent des situations d'exploitation liées à la traite dans plusieurs secteurs économiques: le bâtiment, l'hôtellerie et la restauration, l'économie domestique et l'agriculture. Cela peut donc concerner autant des femmes, que des
Qu'est-ce que la traite des êtres humains?

La traite d'êtres humains consiste à recruter des personnes, à les transférer, à les entremettre par le biais d'intermédiaires, à les héberger ou à les accueillir en vue de leur exploitation. Les victimes subissent des contraintes par des moyens illégaux, notamment par l'exploitation de leur détresse et l'exercice de la violence physique et psychique à leur égard. L'exploitation peut recouvrir l'exploitation sexuelle et l'exploitation de la force de travail. Les victimes sont traitées comme de la marchandise et perdent tout droit à l'autodétermination.

hommes, des adultes que des mineurs. Il s'agit de personnes vulnérables, pauvres dans leur pays d'origine, qui sont prêtes à subir le pire pour espérer une vie meilleure en Suisse. En informant la victime et en reconnaissant sa condition, vous pouvez déjà changer le cours de sa vie.

\section{Crédit photo}

(c) Michael Philipp, luxs 